\title{
Scheduling Low Latency Traffic for Wireless Control Systems in 5G Networks
}

\author{
Mark Eisen $^{\dagger} \quad$ Mohammad M. Rashid ${ }^{\dagger}$
}

Alejandro Ribeiro* Dave Cavalcanti ${ }^{\dagger}$

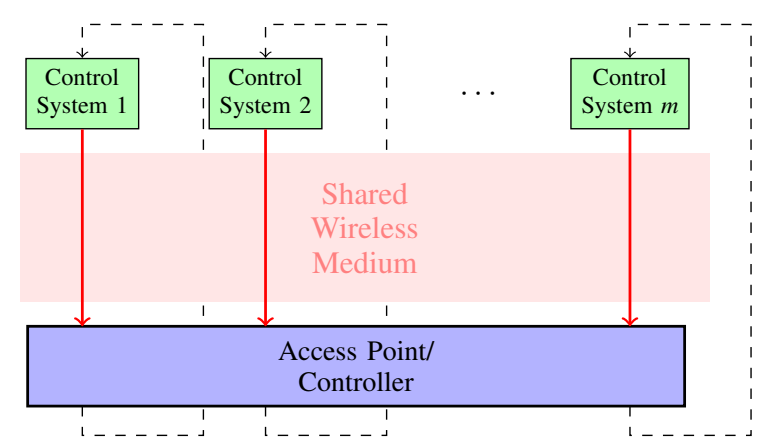

Fig. 1: Wireless control system with $m$ independent systems. Each system contains a sensor that measure state information, which is transmitted to the controller over a wireless channel. The state information is used by the controller to determine control policies for each of the systems. The communication is assumed to be wireless in the uplink and ideal in the downlink.

Specifically in the context of wireless control systems, dynamic schedulers use control system information to provide access to the communication medium dynamically at each step. Resulting "control-aware" scheduling approaches make decisions explicitly based on current control system states [13]-[16] or wireless channel conditions [17], [18]. These works, however, do not consider the low-latency requirements of industrial or IoT systems. A control-aware scheduling design for low-latency systems has previously been developed for next-generation WiFi [19] but has not yet been considered in cellular systems.

In this paper, we propose a scheduling framework for lowlatency control system traffic in 5G communication systems that specifically adapts to the underlying control system needs. We model the switched system dynamics of a wireless control system and use this to model a control-optimal scheduling problem. Using just a simple separation of low-latency traffic for wireless control systems and standard broadband coexisting in the network-i.e. "time" slicing-we demonstrate how control-awareness allows us to effectively schedule lowlatency traffic in just a single subframe. Numerical results demonstrate the benefit of control-optimal scheduling over standard throughput-based scheduling design.

\section{Problem Formulation}

Supported by Intel Science and Technology Center for Wireless $\mathrm{Au}$ tonomous Systems. The authors are with †'Intel Corporation, Hillsboro, OR 97124 and *University of Pennsylvania, Philadelphia, PA 19104. Emails: \{mark.eisen, mamun.rashid, dave.cavalcanti\}@intel.com and \{aribeiro\}@seas.upenn.edu.
Consider a system of $m$ independent control systems as show in Figure 1, where each system $i=1, \ldots, m$ maintains a state variable $\mathbf{x}_{i} \in \mathbb{R}^{p}$. The dynamics evolve over a discrete 
time index $k$. Given a control input $\mathbf{u}_{i, k} \in \mathbb{R}^{q}$, the state evolve based on the generic linear state space representation,

$$
\mathbf{x}_{i, k+1}=\mathbf{A}_{i} \mathbf{x}_{i, k}+\mathbf{B}_{i} \mathbf{u}_{i, k}+\mathbf{w}_{k}
$$

where $\mathbf{A}_{i} \in \mathbb{R}^{p \times p}$ and $\mathbf{B}_{i} \in \mathbb{R}^{p \times q}$ are matrices that define the system dynamics, and $\mathbf{w}_{k} \in \mathbb{R}^{p}$ is i.i.d. random noise with zero mean and co-variance $\mathbf{W}_{i}$ that captures the noise in the model. The plant dynamics in (1) is itself generic and can represent different systems found in IoT environments. Systems that have nonlinear dynamics can be modeled as approximately linear close to equilibrium points, with linearization error captured by $\mathbf{w}_{k}$.

In modern IoT settings, such plants represent a set of devices, e.g. machinery or robotic systems, whose state is measured locally on-device but whose control signal input $\mathbf{u}_{i, k}$ is computed by a centralized processor/coordinator. This so-called control loop is thus closed through wireless communication between the plants and the centralized controller co-located at a common wireless $5 \mathrm{G}$ base station (BS). The dynamics are modeled here as discrete, but in reality the plants evolve continuously over time. With fast dynamics and high sampling rates, many IoT devices require strict low latency between the time state information is measured by the plant to when it can be processed by the controller at the BS. Observe in Figure 1 that this model restricts its attention to wireless connections in uplink of the control loop, while downlink is assumed to occur over an ideal channel.

Aside from latency requirements, wireless communications is inherently noisy and prone to packet drops. Thus, to model the closed-loop dynamics of the plants, we must consider the effect of lost state information. To study the details, consider a generic linear control $\mathbf{u}_{i, k}=\mathbf{K}_{i} \mathbf{x}_{i, k}$ for some matrix $\mathbf{K}_{i} \in$ $\mathbb{R}^{q \times p}$ that drives the state $\mathbf{x}_{i, k}$ to a desired operating point. When exact state information is not known to the BS due to packet drops, it estimates the state of device $i$ at time $k$ as

$$
\hat{\mathbf{x}}_{i, k}^{\left(l_{i, k}\right)}:=\left(\mathbf{A}_{i}+\mathbf{B}_{i} \mathbf{K}_{i}\right)^{l_{i, k}} \mathbf{x}_{i, k-l_{i, k}},
$$

where $l_{i, k} \geq 1$ is a counter of successive failed transmissions. Observe that in (2) we assume the BS/controller has knowledge of $\mathbf{A}_{i}, \mathbf{B}_{i}$, and $\mathbf{K}_{i}$, but not the noise $\mathbf{w}_{k}$.

At time $k$, if the state information is received, the controller applies the input $\mathbf{u}_{i, k}=\mathbf{K}_{i} \mathbf{x}_{i, k}$ using the exact state, and otherwise applies input $\mathbf{u}_{i, k}=\mathbf{K}_{i} \hat{\mathbf{x}}_{i, k}$. At each cycle $k$ we consider a binary variable $\gamma_{i, k} \in\{0,1\}$ that indicates whether the uplink transmission was successful. We obtain then the following switched system dynamics for $\mathbf{x}_{i, k}$ as

$$
\mathbf{x}_{i, k+1}= \begin{cases}\left(\mathbf{A}_{i}+\mathbf{B}_{i} \mathbf{K}_{i}\right) \mathbf{x}_{i, k}+\mathbf{w}_{k}, & \gamma_{i, k}=1 \\ \mathbf{A}_{i} \mathbf{x}_{i, k}+\mathbf{B}_{i} \mathbf{K}_{i} \hat{\mathbf{x}}_{i, k}^{\left(l_{i, k}\right)}+\mathbf{w}_{k}, & \gamma_{i, k}=0\end{cases}
$$

The previous transmission counter $l_{i, k}$ is updated at time $k$ as

$$
l_{i, k+1}= \begin{cases}1, & \gamma_{i, k}=1, \\ l_{i, k}+1, & \gamma_{i, k}=0 .\end{cases}
$$

Observe that the successive error between the true and estimated state can be written as $\mathbf{e}_{i, k}:=\mathbf{x}_{i, k}-\hat{\mathbf{x}}_{i, k}^{\left(l_{i, k}\right)}=$

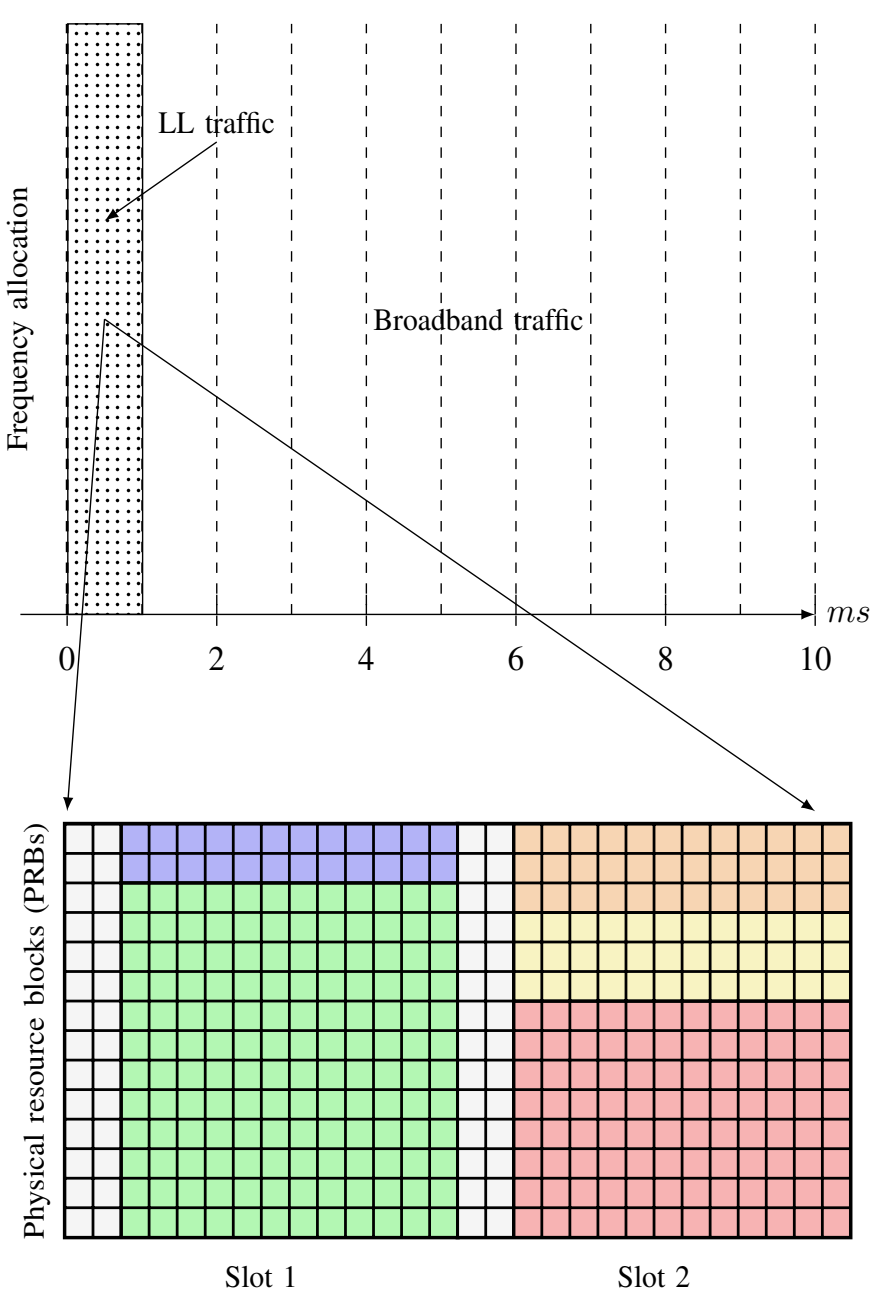

Fig. 2: 5G time-sliced frame structure (top), consisting of 10 equal sized subframes of length $1 \mathrm{~ms}$. A single subframe of each frame (dotted) is reserved for low-latency (LL) control system traffic. Within this subframe (bottom), users are scheduled into time-divided slots and frequency divided subcarriers - the number of each determined by a given numerology. In the presented example, transmissions of $m=5$ plants (green, blue, red, yellow, orange) across $s=2$ slots and $b=14$ PRBs. Each slot contains 14 OFDM symbols, with the first two reserved for control overhead (gray).

$\sum_{j=0}^{l_{i, k}-1}\left(\mathbf{A}_{i}+\mathbf{B}_{i} \mathbf{K}_{i}\right)^{j} \mathbf{w}_{i, k-j-1}$. It is evident that this error grows with the transmission counter $l_{i, k}$. We proceed now to describe the $5 \mathrm{G}$ scheduling architecture and a framework for scheduling low-latency traffic to control IoT devices.

\section{A. 5G scheduling architecture}

The $5 \mathrm{G}$ scheduling framework provides a flexible two dimensional grid-like architecture for scheduling transmissions. Such transmissions are slotted into a continuous series of frames - each of which consists of 10 equally sized subframes of length $1 \mathrm{~ms}$. Each subframe moreover consists of 14 OFDM symbols, two of which we assume are reserved for overheadsee, e.g., [20], [21]. In modern IoT systems, standard broad- 
band wireless traffic is mixed with transmissions associated with control systems fast dynamics or time critical features. This latter traffic has strict latency and reliability requirements that make it incompatible with standard broadband scheduling methods. Various features of $5 \mathrm{G}$, such as network slicing [11] and puncturing [12], have been proposed to meet service requirements of low latency traffic, requiring varying degrees of complexity. Given the periodic nature of low-latency control system transmissions, we propose a simple separation of broadband and low-latency traffic by subframe-i.e. a timedomain slicing of low latency and broadband traffic. E.g., with a $10 \mathrm{~ms}$ sampling rate, we may reserve one or two subframes out of every frame for the scheduling of low-latency traffic while leaving the network free for broadband traffic during the remaining time. This free time may also be used for both control signal overhead in scheduling as well as computational effort in determining optimal schedules.

For the purposes of this paper and without loss of generality, we thus restrict our attention to a single subframe for the scheduling of low-latency traffic in every 10 millisecond long frame. Such an architecture ensures a 1 millisecond latency bound on the uplink of state information to the controller-a necessary requirement for many industrial control systems [1]. Within each subframe, the specific grid size is determined by a given numerology. A specified numerology determines both the number $s$ of time-divided slots - each of length $t_{s}:=1 / s$ milliseconds—and $b$ physical resource blocks (PRBs) - each of bandwidth $f_{b}^{\prime}:=12 f_{b}$, where $f_{b}$ is the so-called subcarrier spacing (SCS) of the given numerology. A higher index numerology will have more, shorter length slots and fewer, larger sized PRBs. We refer the reader to [7], [22] for more details on the $5 \mathrm{G}$ frame structure.

In addition to an assignment of slots and PRBs, each transmission is given a modulation and coding scheme (MCS). For user $i$, its MCS $\mu_{i}$ is selected from a discrete set of values $\mathcal{M}:=\{0,1, \ldots, \eta\}$. A higher MCS value indicates a higher modulation order and coding rate, which will generally increase the speed of transmission, or data rate, but with higher probability of packet error. To quantify these effects, we further consider a fading channel state, or effective SNR, vector for user $i$ at cycle $k$ as $\mathbf{h}_{i, k} \in \mathbb{R}_{+}^{b}$, where $\mathbf{h}_{i, k}(j)$ is the fading channel gain in PRB $j$. We assume the channel coherence time is such that this state varies across control cycles but not within a single cycle. This is to say that $\mathbf{h}_{i, k}$ is constant within a single $5 \mathrm{G}$ frame. Given an MCS $\mu$ and channel state $\mathbf{h}$, we first define a function $q(\mu, \mathbf{h})$ which returns the probability of packet loss, otherwise called packet error rate (PER). Given the switched control dynamics in (3), we equivalently say that $\gamma_{i, k}=0$ with probability $q\left(\mu_{i, k}, \mathbf{h}_{i, k}\right)$. Likewise, we define $\tau(\mu, B)$ to be a function that, given an MCS $\mu$ and bandwidth $B$, returns the maximum time taken for a single transmission attempt. Both of these functions play a critical role in determining scheduling decisions in time-sensitive wireless control system settings. We are, in particular, interested in exploring the trade-off between PER and transmission time with different slot, PRB, and MCS selections. Generally speaking, the functions $q(\mu, \mathbf{h})$ and $\tau(\mu, B)$ relate to $\mu$ by

$$
\mu^{\prime}>\mu \Longrightarrow q(\mu, \mathbf{h}) \leq q\left(\mu^{\prime}, \mathbf{h}\right), \quad \tau\left(\mu^{\prime}, B\right) \leq \tau(\mu, B)
$$

\section{Optimal Scheduling}

In each frame/control cycle, we are interested in scheduling the low-latency transmissions of plant states to the BS such as to maximize the performance of the system utilizing the time-sliced 5G framework in Fig. 2-that is, scheduling low-latency control system uplinks in a single subframe of length $1 \mathrm{~ms}$. The varying nature of both channel and plant states necessitates that the optimal scheduling decision be adaptive to current conditions of the system. For notation convenience, we define the collection of all plant states $\mathbf{X}_{k}:=\left[\mathbf{x}_{1, k} ; \ldots ; \mathbf{x}_{m, k}\right] \in \mathbb{R}^{m \times p}$ and channels $\mathbf{H}_{k}:=$ $\left[\mathbf{h}_{1, k} ; \ldots ; \mathbf{h}_{m, k}\right] \in \mathbb{R}_{++}^{m \times b}$ at cycle $k$.

To specify a scheduling decision, recall that a subframe can be divided as a $s \times b$ grid, with $s$ time slots and $b$ frequency resource blocks. Each plant may be scheduled in any set of adjacent grid points of the sub-frame; we denote this allocation for plant $i$ at cycle $k$ by a binary matrix $\mathbf{A}_{i, k}=\{0,1\}^{s \times b}$, where $\mathbf{A}_{i, k}=\mathbf{0}$ indicates no transmission. We further specify the set of grids with adjacent allocations as $\mathcal{A}$ and denote by $l_{i, k}$ and $n_{i}$ the number of adjacent allocations in the horizontal (time-wise) and vertical (frequency-wise) directions, respectively. The lengths can equivalently be seen respectively as the total time and bandwidth of the scheduling allocation. To avoid collision, we impose that $\sum_{i} \mathbf{A}_{i, k} \leq \mathbf{1 1}^{T}$-at most one user may be assigned to any grid point.

Furthermore, each transmission is given an MCS allocation $\mu_{i, k}$ that ultimately determines both its speed and probability of packet loss. Recalling the effect and MCS $\mu$ has on these metrics in (5) and given the fixed size of a single slot- $t_{s}-$ and fixed size of PRBs- $f_{b}^{\prime}$-it reasons to select the smallest $\mu_{i, k}$ such that the transmission time is completed by the end of its assigned slots; this ensures the smallest possible packet error rate. The MCS $\mu_{i}$ is then fully determined by its timeand frequency-wise allocation lengths $l_{i, k}$ and $n_{i}$ as

$$
\mu_{i, k}:=\mu\left(\mathbf{A}_{i, k}\right)=\min \left\{\mu \in \mathcal{M} \mid \tau\left(\mu, n_{i} f_{b}^{\prime}\right) \leq l_{i, k} t_{s}\right\} .
$$

The optimal schedule is one that minimizes some cost or performance metric. Standard schedulers are ultimately interested in some measure of throughput. A natural cost to promote throughput is the expected number of successful transmissions per cycle. Given a set of scheduling assignments $\mathbb{A}=\left\{\mathbf{A}_{i}\right\}_{i=1}^{m}$, we may write this cost as

$$
\omega(\mathbb{A}, \mathbf{H}):=\mathbb{E}_{\mathbf{H}} \sum_{i} \mathbb{1}\left[\gamma_{i}=0\right]=\sum_{i=1}^{m} q\left(\mu\left(\mathbf{A}_{i}\right), \mathbf{h}_{i}\right) .
$$

The optimal scheduling at cycle $k$ in the given $5 \mathrm{G}$ framework can be written as the solution to the problem

$$
\begin{array}{r}
\left\{\mathbf{A}_{1, k}, \ldots, \mathbf{A}_{m, k}\right\}:=\mathbb{A}^{*}\left(\mathbf{H}_{k}\right)=\underset{\mathbf{A}_{i}, \ldots, \mathbf{A}_{m} \in \mathcal{A}}{\operatorname{argmin}} \omega\left(\mathbb{A}, \mathbf{H}_{k}\right), \\
\text { s.t. } \sum_{i=1}^{m} \mathbf{A}_{i} \leq \mathbf{1 1}^{T} .
\end{array}
$$


In (8), we minimize the cost in (7) under current channel conditions $\mathbf{H}_{k}$, subject to a constraint that restricts a single user to be scheduled in each slot or PRB. The problem in (8) is generally hard to solve, due to the discrete and combinatorial sized search space. However, if we restrict the scheduling assignments to a single grid point-i.e. each user is given a single slot and a fixed number $\rho \mathrm{PRBs}$ - then (8) reduces to a standard linear-sum assignment problem with $n:=s\lfloor b / \rho\rfloor$ distinct and mutually exclusive assignments, each incurring a cost of $q\left(\mu\left(\mathbf{A}_{i}\right), \mathbf{h}_{i}\right)$ In this case, the solution $\mathbb{A}^{*}\left(\mathbf{H}_{k}\right)$ can be found using the Hungarian method [23], or a low complexity greedy approximation [24]-[26]. Thus, at every cycle, given the fading channel states $\mathbf{H}_{k}$, the optimal schedule is found by determining the slot/PRB assignments $\left\{\mathbf{A}_{1, k}, \ldots, \mathbf{A}_{m, k}\right\}$ via (8). The MCS selections then follow from (6).

The optimal schedules found via (8) may not, in practice, be enough to obtain strong performance over the tight latency constraints required. By limiting to a single subframe we are placing a potentially severe restriction on the number of plants that can be serviced in each cycle. As modern IoT system grow in size, the more traditional utility measure of (7) is not the most appropriate to adequately utilize the limited radio resources available. Observe that the optimal design problem (8) seeks to maximize the total number of successful transmission across the systems, but agnostic to the control plant states and dynamics. Thus, it does not capture any sense of priority or urgency with regard to certain plants over others. Indeed, an important insight to consider is the fact that we are ultimately interested in the performance of the control systems; the performance of the underlying communications network is only of interest in as far as it effects the plant dynamics in (3). We proceed in the next section to derive a more accurate utility and optimal scheduling design problem for $5 \mathrm{G}$ architectures.

\section{A. Control optimal scheduling}

Incorporating the switch system plant dynamics in (3) and standard measure of control performance, we may construct a control-aware variation of the optimal scheduling problem in (8). Consider the standard Lyapunov quadratic cost function $L(\mathbf{x}):=\mathbf{x}^{T} \mathbf{P} \mathbf{x}$ for a given positive definite matrix $\mathbf{P} \in \mathbb{R}^{p \times p}$. The quadratic cost $L(\mathbf{x})$ measures the current cost of a control system in state $\mathbf{x}-$ e.g. if $\mathbf{x}$ represents some distance from desired operating point, we may set $\mathbf{P}=\mathbf{I}$ and have $L(\mathbf{x})$ reflect the Euclidean norm. We are then, after all, interested in designing $5 \mathrm{G}$ scheduling policies that achieve good performance across all systems, which can be measured with as the $\operatorname{sum} \sum_{i} L\left(\mathbf{x}_{i, k}\right.$ at time $k$.

From here, we show how a scheduling decision impacts the control performance of each plant. Wireless communications are subject to random packet drops given the channel state, which ultimately effect the dynamic evolution of the plants. Recall the packet error rate experience by system $i$ given a scheduling assignment $\mathbf{A}_{i}$ and channel state $\mathbf{h}_{i}$ given by $q\left(\mu\left(\mathbf{A}_{i}\right), \mathbf{h}_{i}\right)$. Using the two cases of dynamics given in (3), we may consider the expected quadratic cost under such a packet error rate given the known or estimated state $\hat{\mathbf{x}}_{i, k}^{\left(l_{i, k}\right)}$ as

$$
\begin{aligned}
& \mathbb{E}_{\mathbf{q}, \mathbf{w}}\left\{L\left(\mathbf{x}_{i, k+1}\right) \mid \mathbf{A}_{i}, \mathbf{h}_{i}, \hat{\mathbf{x}}_{i, k}^{\left(l_{i, k}\right)}\right\}= \\
& \mathbb{E}_{\mathbf{w}}\left\{\left(1-q\left(\mu\left(\mathbf{A}_{i}\right), \mathbf{h}_{i}\right)\right) L\left(\left(\mathbf{A}_{i}+\mathbf{B}_{i} \mathbf{K}_{i}\right) \mathbf{x}_{i, k}+\mathbf{w}_{k}\right)\right. \\
& \left.\quad+q\left(\mu\left(\mathbf{A}_{i}\right), \mathbf{h}_{i}\right) L\left(\mathbf{A}_{i} \mathbf{x}_{i, k}+\mathbf{B}_{i} \mathbf{K}_{i} \hat{\mathbf{x}}_{i, k}^{\left(l_{i, k}\right)}+\mathbf{w}_{k}\right)\right\}
\end{aligned}
$$

The expectation in the left hand side of (9) is with respect to the random packet loss and system noise $\mathbf{w}$, while the expectation in the right hand side is only with respect to $\mathbf{w}$. Given states $\mathbf{h}_{i}$ and $\hat{\mathbf{x}}_{i, k}^{\left(l_{i, k}\right)}$ and scheduling assignment $\mathbf{A}_{i}$, the expected cost at the next step is a convex combination of the two possible evolutions of $\mathbf{x}_{i}$ under such a scheduling. We may further reduce the cost in 90 taking the expectation over $\mathbf{w}_{k}$ and reducing as

$$
\begin{aligned}
& \mathbb{E}_{\mathbf{q}, \mathbf{w}}\left\{L\left(\mathbf{x}_{i, k+1}\right) \mid \mathbf{A}_{i}, \mathbf{h}_{i}, \hat{\mathbf{x}}_{i, k}^{\left(l_{i, k}\right)}\right\}= \\
& {\left[\left\|\mathbf{A}_{i}^{c} \hat{\mathbf{x}}\right\|_{\mathbf{P}^{\frac{1}{2}}}^{2}+\operatorname{Tr}(\mathbf{P} \mathbf{W})+\sum_{j=1}^{l} \omega_{i}^{j}\right]+} \\
& q\left(\mu\left(\mathbf{A}_{i}\right), \mathbf{h}_{i}\right) \sum_{j=0}^{l_{i, k}-1}\left[\operatorname{Tr}\left(\mathbf{A}_{i}^{c T}\left(\mathbf{A}_{i}^{T} \mathbf{P}^{\frac{1}{j}} \mathbf{A}_{i}\right)^{j} \mathbf{A}_{i}^{c} \mathbf{W}\right)-\omega_{i}^{j+1}\right],
\end{aligned}
$$

where $\mathbf{A}_{i}^{c}:=\mathbf{A}_{i}+\mathbf{B}_{i} \mathbf{K}_{i}$ and $\omega_{i}^{j}:=\operatorname{Tr}\left[\left(\mathbf{A}_{i}^{T} \mathbf{P}^{1 / j} \mathbf{A}_{i}\right)^{j} \mathbf{W}\right]$ are defined for notational convenience. We point out that algebraic details of the reduction from (9) to (10) can be found in the proof of [19. Proposition 1].

The complete reduced form of the expected control cost in (10) reveals how the control-aware scheduling problem may be formulated. Firstly, observe that the effect of a scheduling assignment $\mathbf{A}_{i}$ through the packet error rate $q\left(\mu\left(\mathbf{A}_{i}\right), \mathbf{h}_{i}\right)$ seen in the rightmost term in 10 is not impacted by the estimated state value $\hat{\mathbf{x}}_{i, k}^{\left(l_{i, k}\right)}$. This implies that a scheduling decision does not require any estimation of the state, and only needs to know the counter $l_{i, k}$ of the last successful transmission. Thus, by removing the first term in the right hand side of (10) that does not depend upon $\hat{\mathbf{x}}_{i, k}^{\left(l_{i, k}\right)}$, we may consider the a cost of a complete scheduling $\mathbb{A}$ that sums the expected costs across all systems given channel conditions $\mathbf{H}$ and transmission counters $\mathbf{l}_{k}:=\left[l_{1, k} ; \ldots ; l_{m, k}\right]$ as

$$
\omega^{\prime}\left(\mathbb{A}, \mathbf{H}, \mathbf{l}_{k}\right):=\sum_{i=1}^{m} c_{i}\left(l_{i, k}\right) q\left(\mu\left(\mathbf{A}_{i}\right), \mathbf{h}_{i}\right)
$$

where we have defined the control system weighting term for system $i$ as

$$
c_{i}\left(l_{i, k}\right):=\sum_{j=0}^{l_{i, k}-1}\left[\operatorname{Tr}\left(\mathbf{A}_{i}^{c T}\left(\mathbf{A}_{i}^{T} \mathbf{P}^{\frac{1}{j}} \mathbf{A}_{i}\right)^{j} \mathbf{A}_{i}^{c} \mathbf{W}\right)-\omega_{i}^{j+1}\right] .
$$


The control optimal scheduling at cycle $k$ in the given $5 \mathrm{G}$ framework can then be written as the solution to the problem

$$
\begin{array}{r}
\left\{\mathbf{A}_{1, k}, \ldots, \mathbf{A}_{m, k}\right\}:=\underset{\mathbf{A}_{i}, \ldots, \mathbf{A}_{m} \in \mathcal{A}}{\operatorname{argmin}} \omega^{\prime}\left(\mathbb{A}, \mathbf{H}_{k}, \mathbf{l}_{k}\right), \\
\text { s.t. } \quad \sum_{i=1}^{m} \mathbf{A}_{i} \leq \mathbf{1 1}^{T} .
\end{array}
$$

In (13) the scheduling allocation is determined so as to minimize the summation of the expected quadratic costs in (9) for all plants $i=1, \ldots, m$. This reduces to a linear scaling of the packet delivery rate $q\left(\mu\left(\mathbf{A}_{i}\right), \mathbf{h}_{i}\right)$ by a weighting term $c_{i}\left(l_{i, k}\right)<0$ for each system $i$, that takes into accounts the dynamics of plant $i$ and its last transmission counter $l_{i, k}$. As in the previous case, when restricting allocation to a single slot and $\rho$ PRBs for each user, (13) becomes a standard linear cost assignment problem whose solution can be found using the Hungarian method [23] or a low complexity greedy approximation [24]-[26]. Note that, when $m>n:=s\lfloor b / \rho\rfloor$, not all users can be scheduled in each frame.

The full scheduling algorithm can be summarized as follows. At each cycle $k$, the plant states are sampled locally at each device to be transmitted. In the current $5 \mathrm{G}$ transmission frame, we reserve a single subframes of length $1 \mathrm{~ms}$ to schedule for a total of $n=s\lfloor b / \rho\rfloor$ scheduling assignment blocks. The scheduling decision is then made with the routine:

1) Measure channel states $\mathbf{H}_{k}=\left[\mathbf{h}_{1, k} ; \ldots ; \mathbf{h}_{m, k}\right] \in \mathbb{R}_{++}^{m \times b}$.

2) Determine scheduling alloc. $\left\{\mathbf{A}_{1, k}, \ldots, \mathbf{A}_{m, k}\right\}$ via (13).

3) Set MCS values $\mu_{i, k}$ via (6) for all $i=1, \ldots, m$.

4) Device $i$ transmits with packet success $\gamma_{i, k}=1$ w.p. $q\left(\mu_{i, k}, \mathbf{h}_{i}\right)$ for all $i=1, \ldots, m$.

5) $\mathrm{BS}$ applies corresponding control and plant $i$ evolves via (3) for all $i=1, \ldots, m$.

6) Counter $l_{i, k}$ updates via (4) for all $i=1, \ldots, m$.

\section{Simulation Results}

To evaluate the performance of the control-optimal scheduling procedure outlined in the previous section, we perform numerical simulations on the low latency control problem of maintaining a series of inverted pendulums on a horizontal cart over a wireless channel. The highly unstable dynamics of the inverted pendulum make it a representative example of control system that requires fast control cycles, and subsequently low-latency communications when being controlled over a wireless medium. Consider a series of $m$ identical inverted pendulums - where each pendulum is attached at one end to a cart that can move along a single, horizontal axis-using the modeling of the inverted pendulum. The state is $p=4$ dimensional vector that maintains the position and velocity of the cart along the horizontal axis, and the angular position and velocity of the pendulum, i.e. $\mathbf{x}_{i, k}:=\left[x_{i, k}, \dot{x}_{i, k}, \theta_{i, k}, \dot{\theta}_{i, k}\right]$. The system input $u_{i, k}$ reflects a horizontal force placed upon $i$ th pendulum. By applying a zeroth order hold on the continuous dynamics with a state sampling rate of 10 milliseconds and linearizing, we obtained the following discrete linear dynamic matrices of the pendulum system $\mathbf{A}_{i}$ and $\mathbf{B}_{i}$. Because the
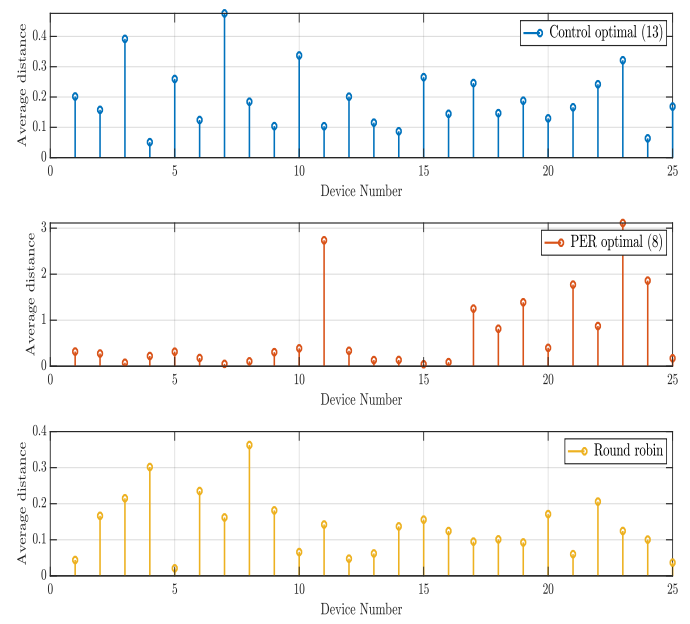

Fig. 3: Average distances from center vertical for $m=25$ pendulums using different scheduling methods for a single trial. The control-optimal scheduling and round robin keep the pendulums while the PER optimal scheduling does not.

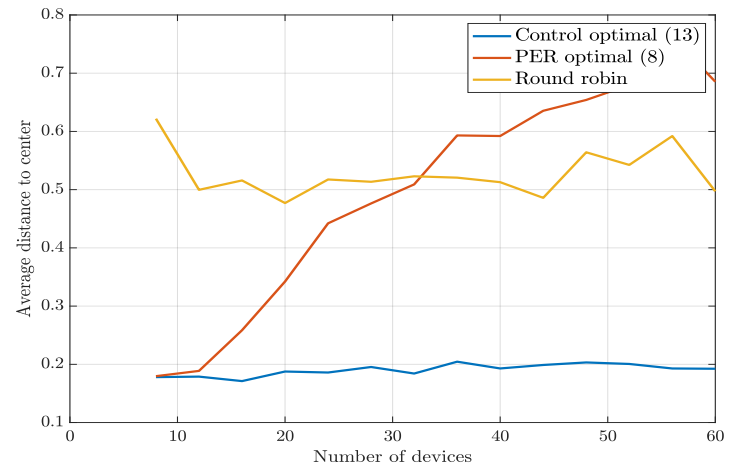

Fig. 4: The average distances from center vertical for increasing number of pendulums over 10 independent trials. The control-optimal scheduling maintains a small distance from center up to 60 total devices, while the PER optimal scheduling gets worse as system size grows.

state $\mathbf{x}_{i, k}$ measures the angle of the $i$ th pendulum at time $k$, the goal is to keep this close to zero, signifying that the pendulum remains upright. We perform the scheduling using the proposed control-optimal formulation in (13), the packet error rate (PER)-optimal scheduling formulation (8), and a baseline round robin scheduling procedure. Each simulation is run for a total of 1000 seconds using an LQR controller. Our simulation environment consists of a $20 \mathrm{MHz}$ channel with devices dropped in a $50 \mathrm{~m}$ radius from the BS. The numerology includes 2 slots of length $500 \mu$ s and a subcarrier spacing of $30 \mathrm{kHz}$, with each user allocated $n=12$ PRBs.

In Figure 4, we show the average distance of each of $m=25$ pendulums in a trial simulation using each of the three scheduling methods. Both the control-optimal scheduling and the round robin keep the pendulums upright, while the 
PER-optimal scheduling is not able to keep all the pendulums. For a more comprehensive view of the performance, we perform simulations with an increasing number of devices with multiple trials. In Figure 4 we show the average distance across 10 independent trials to the center across all the pendulums for increasing number of total devices sharing the $5 \mathrm{G}$ network. As can be seen, as the number of devices increase, the PER optimal schedule has a harder time keep the pendulums close to the center, while the control-optimal scheduling maintains a steady distance from the center even as the number of devices increase. The round robin scheduler is consistent across network size but cannot keep the pendulums close to center. This demonstrates that, in the control optimal scheduling, the consideration of the pendulum dynamics and the control state helps make stronger scheduling decisions in keeping the systems in good condition.

\section{CONCLUSION}

In this paper we develop a control-optimal approach towards scheduling for low-latency, or time sensitive, wireless control systems in 5G networks. Because many control systems in industrial settings require very low latency transmission to operate effectively, there is an intrinsic challenge in trading off the data rates necessary to achieve low latency with the packet error rates necessary for high reliability. We propose a simple time-slicing architecture that reserves a single or couple of subframes in each $5 \mathrm{G}$ frame for the scheduling of low-latency traffic used for the control systems. We further demonstrate that by allocating radio resources relative to underlying control system dynamics and states, we can more effectively utilize the limited scheduling resources available in the available subframes in low-latency $5 \mathrm{G}$ systems. Numerical results demonstrate stronger performance relative to controlagnostic scheduling in terms of the number of system that can be supported by the communication network.

\section{REFERENCES}

[1] Anitha Varghese and Deepaknath Tandur, "Wireless requirements and challenges in industry 4.0," in Contemporary Computing and Informatics (IC3I), 2014 International Conference on. IEEE, 2014, pp. 634-638.

[2] Xiaomin Li, Di Li, Jiafu Wan, Athanasios V Vasilakos, Chin-Feng Lai, and Shiyong Wang, "A review of industrial wireless networks in the context of industry 4.0," Wireless networks, vol. 23, no. 1, pp. 23-41, 2017.

[3] Martin Wollschlaeger, Thilo Sauter, and Juergen Jasperneite, "The future of industrial communication: Automation networks in the era of the internet of things and industry 4.0," IEEE Industrial Electronics Magazine, vol. 11, no. 1, pp. 17-27, 2017.

[4] Chengjie Wu, Mo Sha, Dolvara Gunatilaka, Abusayeed Saifullah, Chenyang Lu, and Yixin Chen, "Analysis of edf scheduling for wireless sensor-actuator networks," in Quality of Service (IWQoS), 2014 IEEE 22nd International Symposium of. IEEE, 2014, pp. 31-40.

[5] Songwu Lu, Vaduvur Bharghavan, and Rayadurgam Srikant, "Fair scheduling in wireless packet networks," IEEE/ACM Transactions on networking, vol. 7, no. 4, pp. 473-489, 1999.

[6] Matthew Andrews, Krishnan Kumaran, Kavita Ramanan, Alexander Stolyar, Phil Whiting, and Rajiv Vijayakumar, "Providing quality of service over a shared wireless link," IEEE Communications magazine, vol. 39, no. 2, pp. 150-154, 2001.

[7] Stefan Parkvall, Erik Dahlman, Anders Furuskar, and Mattias Frenne, "NR: The new 5G radio access technology," IEEE Communications Standards Magazine, vol. 1, no. 4, pp. 24-30, 2017.
[8] Bikramjit Singh, Zexian Li, Olav Tirkkonen, Mikko A Uusitalo, and Preben Mogensen, "Ultra-reliable communication in a factory environment for $5 \mathrm{~g}$ wireless networks: Link level and deployment study," in 2016 IEEE 27th Annual International Symposium on Personal, Indoor, and Mobile Radio Communications (PIMRC). IEEE, 2016, pp. 1-5.

[9] Nurul H Mahmood, Mads Lauridsen, Gilberto Berardinelli, Davide Catania, and Preben Mogensen, "Radio resource management techniques for eMBB and mMTC services in 5G dense small cell scenarios," in 2016 IEEE 84th Vehicular Technology Conference (VTC-Fall). IEEE, 2016, pp. 1-5.

[10] Chih-Ping Li, Jing Jiang, Wanshi Chen, Tingfang Ji, and John Smee, "5G ultra-reliable and low-latency systems design," in 2017 European Conference on Networks and Communications (EuCNC). IEEE, 2017, pp. $1-5$.

[11] Petar Popovski, Kasper Fløe Trillingsgaard, Osvaldo Simeone, and Giuseppe Durisi, "5G wireless network slicing for eMBB, URLLC, and mMTC: A communication-theoretic view," IEEE Access, vol. 6, pp. 55765-55779, 2018.

[12] Arjun Anand, Gustavo De Veciana, and Sanjay Shakkottai, "Joint scheduling of URLLC and eMBB traffic in 5G wireless networks," in IEEE INFOCOM 2018-IEEE Conference on Computer Communications. IEEE, 2018, pp. 1970-1978.

[13] Anton Cervin and Toivo Henningsson, "Scheduling of event-triggered controllers on a shared network," in Proc. of the 47th IEEE Conf. on Dec. and Control (CDC), 2008, pp. 3601-3606.

[14] Mohammad H Mamduhi, Domagoj Tolić, Adam Molin, and Sandra Hirche, "Event-triggered scheduling for stochastic multi-loop networked control systems with packet dropouts," in Decision and Control (CDC), 2014 IEEE 53rd Annual Conference on. IEEE, 2014, pp. 2776-2782.

[15] Ling Shi, Peng Cheng, and Jiming Chen, "Optimal periodic sensor scheduling with limited resources," IEEE Transactions on Automatic Control, vol. 56, no. 9, pp. 2190-2195, 2011.

[16] Duo Han, Junfeng Wu, Huanshui Zhang, and Ling Shi, "Optimal sensor scheduling for multiple linear dynamical systems," Automatica, vol. 75, pp. 260-270, 2017.

[17] Konstantinos Gatsis, Miroslav Pajic, Alejandro Ribeiro, and George J. Pappas, "Opportunistic control over shared wireless channels," IEEE Transactions on Automatic Control, vol. 60, no. 12, pp. 3140-3155, December 2015.

[18] Yehan Ma, Jianlin Guo, Yebin Wang, Ankush Chakrabarty, Heejin Ahn, Philip Orlik, and Chenyang Lu, "Optimal dynamic scheduling of wireless networked control systems," in Proceedings of the 10th ACM/IEEE International Conference on Cyber-Physical Systems. ACM, 2019, pp. 77-86.

[19] Mark Eisen, Mohammad M Rashid, Konstantinos Gatsis, Dave Cavalcanti, Nageen Himayat, and Alejandro Ribeiro, "Control aware radio resource allocation in low latency wireless control systems," IEEE Internet of Things Journal, vol. 6, no. 5, pp. 7878-7890, 2019.

[20] Preben Mogensen, Kari Pajukoski, Esa Tiirola, Eeva Lähetkangas, Jaakko Vihriälä, Seppo Vesterinen, Matti Laitila, Gilberto Berardinelli, Gustavo WO Da Costa, Luis GU Garcia, et al., "5G small cell optimized radio design," in 2013 IEEE Globecom Workshops (GC Wkshps). IEEE, 2013, pp. 111-116.

[21] Eeva Lähetkangas, Kari Pajukoski, Jaakko Vihriälä, Gilberto Berardinelli, Mads Lauridsen, Esa Tiirola, and Preben Mogensen, "Achieving low latency and energy consumption by 5G TDD mode optimization," in 2014 IEEE International Conference on Communications Workshops (ICC). IEEE, 2014, pp. 1-6.

[22] Mamta Agiwal, Abhishek Roy, and Navrati Saxena, "Next generation $5 \mathrm{~g}$ wireless networks: A comprehensive survey," IEEE Communications Surveys \& Tutorials, vol. 18, no. 3, pp. 1617-1655, 2016.

[23] Harold W Kuhn, "The hungarian method for the assignment problem," Naval research logistics quarterly, vol. 2, no. 1-2, pp. 83-97, 1955.

[24] H Edwin Romeijn and Dolores Romero Morales, "A class of greedy algorithms for the generalized assignment problem," Discrete Applied Mathematics, vol. 103, no. 1-3, pp. 209-235, 2000.

[25] Qinma Kang, Hong He, and Huimin Song, "Task assignment in heterogeneous computing systems using an effective iterated greedy algorithm," Journal of Systems and Software, vol. 84, no. 6, pp. 985992, 2011.

[26] Kiran Thekumparampil, Andrew Thangaraj, and Rahul Vaze, "Combinatorial resource allocation using submodularity of waterfilling," IEEE Transactions on Wireless Communications, vol. 15, no. 1, pp. 206-216, 2015. 